Aim of the study: Recent studies showed relatively better outcome for children with refractory (refAML) and relapsed acute myeloid leukemia (relAML). Treatment of these patients has not been unified within Polish Pediatric Leukemia/Lymphoma Study Group (PPLLSG) so far. The goal of this study is to analyze the results of this therapy performed between 2005-2011.

Material and methods: The outcome data of 16 patients with refAML and 62 with relAML were analyzed retrospectively. Reinduction was usually based on idarubicine, fludarabine and cytarabine with allogenic hematopoietic stem cell transplant (alloHSCT) in 5 refAML and 30 relAML children.

Results: Seventy seven percent relAML patients entered second complete remission (CR2). Five-year OS and disease-free survival (DFS) were estimated at $16 \%$ and $30 \%$. The outcome for patients after alloHSCT in CR2 (63\%) was better than that of those not transplanted (36\%) with 5-year OS of $34 \%$ vs. 2 -year of $7 \%$ and 5 -year DFS of $40 \%$ vs. $12.5 \%$. Second complete remission achievement and alloHSCT were the most significant predictors of better prognosis ( $p=0.000$ and $p=0.024)$. The outcome of refAML children was significantly worse than relAML with first remission (CR1) rate of $33 \%$, OS and DFS of $25 \%$ at 3 years and $53 \%$ at 2 years, respectively. All survivors of refAML were treated with alloHSCT after CR1.

Conclusions: The uniform reinduction regimen of the documented efficacy and subsequent alloHSCT in remission is needed to improve the outcome for ref/relAML children treated within PPLLSG. The focus should be on the future risk-directed both front and second line AML therapy.

Key words: acute myeloid leukemia, relapse, stem cell transplantation, children.

Contemp Oncol (Pozn) 2014; 18 (1): 48-53 DOI: $10.5114 /$ wo.2014.40436

\section{Outcome of refractory and relapsed acute myeloid leukemia in children treated during 2005-2011 - experience of the Polish Pediatric Leukemia/Lymphoma Study Group (PPLLSG)}

\author{
Jolanta Skalska-Sadowska ${ }^{1}$, Jacek Wachowiak ${ }^{1}$ \\ and Polish Pediatric Leukemia/Lymphoma Study Group (PPLLSG): \\ Jolanta Skalska-Sadowska ${ }^{1}$, Jacek Wachowiak' ${ }^{1}$, Olga Zając-Spychała', Izabela \\ Niewiadomska-Wojnałowicz' Danuta Januszkiewicz-Lewandowska ${ }^{1}$, Walentyna \\ Balwierz ${ }^{2}$, Katarzyna Pawińska-Wąsikowska², Jolanta Goździk³ , Alicja Chybicka ${ }^{4}$, \\ Kinga Potocka ${ }^{4}$, Maryna Krawczuk-Rybak ${ }^{5}$, Katarzyna Muszyńska-Rosłan ${ }^{5}$, Elżbieta \\ Adamkiewicz-Drożyńska ${ }^{6}$, Lucyna Maciejka-Kapuścińska ${ }^{6}$, Grażyna Karolczyk \\ Jerzy Kowalczyk ${ }^{8}$, Beata Wójcik ${ }^{8}$, Wanda Badowska ${ }^{9}$, Tomasz Urasiński ${ }^{10}$, Tomasz \\ Ociepa ${ }^{10}$, Michat Matysiak ${ }^{11}$, Barbara Sikorska-Fic ${ }^{11}$, Tomasz Szczepański ${ }^{12}$, Renata \\ Tomaszewska $^{12}$, Grażyna Sobol ${ }^{13}$, Maria Wieczorek ${ }^{14}$, Irena Karpińska-Derda ${ }^{14}$
}

\author{
'Department of Pediatric Hematology, Oncology and Transplantology, University \\ of Medical Sciences, Poznan, Poland \\ 2Department of Pediatric Oncology and Hematology, Jagiellonian University Medical \\ College, Krakow, Poland \\ 'Department of Transplantology, Jagiellonian University Children's Hospital, Krakow, \\ Poland \\ ${ }^{4}$ Department of Pediatric Bone Marrow Transplantation, Hematology and Oncology, \\ Medical University, Wroclaw, Poland \\ Department of Pediatric Hematology and Oncology, Medical University Bialystok, \\ Poland \\ ${ }^{6}$ Department of Pediatric Hematology and Oncology, Medical University Gdansk, Poland \\ ${ }^{7}$ Department of Pediatric Hematology and Oncology, Children Hospital, Kielce, Poland \\ ${ }^{8}$ Department of Pediatric Hematology, Oncology and Transplantology, Medical \\ University, Lublin, Poland \\ ${ }^{9}$ Department of Pediatric Hematology and Oncology, University of Warmia and Mazury, \\ Olsztyn, Poland \\ ${ }^{10} \mathrm{Clinic}$ of Pediatrics, Hematology and Oncology, Pomeranian Medical \\ University, Szczecin, Poland \\ "Department of Pediatric Hematology and Oncology, Medical University Warsaw, \\ Poland \\ ${ }^{12}$ Department of Pediatric Hematology and Oncology, Medical University of Silesia, \\ Zabrze, Poland \\ ${ }^{13}$ Department of Pediatrics, Medical University of Silesia, Katowice, Poland \\ ${ }^{14}$ Department of Pediatric Hematology and Oncology, Pediatric Center Chorzow, Poland
}

\section{Introduction}

Childhood acute myeloid leukemia (AML) is resistant to therapy (refAML) in $5-10 \%$ or recurs (relAML) in $30-40 \%$ of patients $[1,2]$. Recent trials have shown a higher cure rate, providing an insight into current therapeutic strategies for these children with poor prognosis $[3,4]$.

Treatment of ref/relAML children within the Polish Pediatric Leukemia/ Lymphoma Study Group (PPLLSG) has not been unified so far. The improved outcome among consecutive BFM AML studies encouraged the PPLLSG to adopt the modified BFM relapsed AML 2001/01 protocol [5]. The objective of this retrospective report is to analyze the outcomes to date in comparison 
with other groups and to establish a benchmark for analysis of future therapy.

\section{Material and methods}

Between 2005 and 2011, 16 children (8 boys, 8 girls) with refAML and 62 children (38 boys, 24 girls) with relAML were treated at PPLLSG institutions.

The median time from the date of the first complete remission (CR1) to relapse was 10 months (range 1-77.4). Forty (64\%) relAML patients had early $(\leq 1$ year) and 22 (35\%) late relapse (> 1 year). Table 1 shows the characteristics of the patients in this cohort.

\section{Reinduction treatment}

Informed consent and ethical approval were obtained before the therapy was performed. There was no single reinduction chemotherapy performed. The management varied according to different institutions. IdaFlag (fludarabine, high-dose cytarabine and granulocyte colony-stimulating factor with addition of idarubicin) was the most common basis of the administered regimens: refAML $n=11$, relAML $n=43$.

After reinduction chemotherapy, 35 (5 refAML, 30 relAML) patients underwent allogeneic hematopoietic stem cell transplantation (alloHSCT): 24 (68\%) from a matched unrelated donor (MD), 5 (14\%) from a matched sibling donor (MSD), 3 (8\%) from a mismatched donor (MMD), 1 (3\%) from a haploidentical donor, and for 2 (6\%) there were no data concerning donor type. The majority of children were conditioned for alloHSCT with the busulfan plus cyclophosphamide-based regimen.

\section{Definitions}

Primary refAML, first relapse $A M L$, and risk group at the initial diagnosis were defined according to the AML-BFM Interimphase 2004 protocol. Early treatment response, postreinduction complete remission and second relapse

Table 1. Characteristics of the disease and the patients with refAML and refAML

\begin{tabular}{|c|c|c|}
\hline & $\begin{array}{l}\text { Primary refractory } \\
\qquad \mathrm{AML}(n=16)\end{array}$ & $\begin{array}{l}\text { First relapse } \\
\text { AML }(n=62)\end{array}$ \\
\hline Age & $\begin{array}{l}\text { 1.2-17.1 years } \\
\text { (median } 12.7 \text { ) }\end{array}$ & $\begin{array}{l}0.1-17.5 \text { years } \\
\text { (median } 10.9 \text { ) }\end{array}$ \\
\hline \multicolumn{3}{|l|}{$\begin{array}{l}\text { Risk group at primary } \\
\text { diagnosis }\end{array}$} \\
\hline SR & & $17(27 \%)$ \\
\hline$H R$ & $16(100 \%)$ & $45(72 \%)$ \\
\hline \multicolumn{3}{|l|}{ Initial protocol } \\
\hline AML-BFM 2004 Interim & 15 & 53 \\
\hline ANLL98 & & 9 \\
\hline \multicolumn{3}{|l|}{$\begin{array}{l}\text { Site of primary disease/ } \\
\text { relapse }\end{array}$} \\
\hline isolated BM & 13 & 43 \\
\hline $\mathrm{BM}+\mathrm{CNS}$ & 2 & 10 \\
\hline $\mathrm{BM}+$ other & 1 & 4 \\
\hline isolated CNS & & 4 \\
\hline other isolated & & 1 \\
\hline
\end{tabular}

were defined according to the Relapsed AML 2002/01 protocol [5].

\section{Statistical analysis}

Surviving patients were censored on the $31^{\text {st }}$ of December 2011. For statistical analysis, the Kaplan-Meier and the log-rank test were used. The Cox proportional-hazards regression model allowed us to determine significant time-dependent factors. Results were considered to be statistically significant if the $p$ value was under 0.05 .

\section{Results}

\section{Primary refAML outcome}

Of 16 children, one patient died before reinduction. Fifteen $(93 \%)$ children underwent reinduction chemotherapy and the early good response rate was $40 \%(n=6)$. Five (33\%) children, including one poor early responder, entered first complete remission.

Of the patients who remitted, one had a relapse 9 days after CR1 and died 3.5 months later. Four remaining patients underwent alloHSCT at a median of 1.5 months (range 1.1-1.9) from CR1.

One of the transplanted children relapsed after MSD alloHSCT. The three remaining grafted patients (2 MD, 1 MSD) were the only survivors at the time of the last follow-up (Table 2).

One child who failed to enter CR1 on second-line chemotherapy received matched unrelated donor (MUD) alloHSCT and died 9 days later of progressive disease. By December 31, 2011, three (18.7\%) of 16 refAML children, including 1 poor early responder, were alive, with a median time from CR1 of 33.2 months (range 14.9-34.2).

\section{Relapse AML outcome}

Four children died before reinduction. Among the 58 patients who received chemotherapy, 37 (63\%) were good early responders with missing data in 14 cases. Second complete remission (CR2) was attained in 44 (75\%) children, including 5 poor early responders. One other patient achieved CR2 after MD alloHSCT performed in relapse, resulting in a CR2 rate of $77 \%(n=45)$. The CR2 rate among children treated initially according to protocols AML-BFM 2004 Interim and ANLL98 was 70\% $(n=35)$ and $88 \%(n=8)$ respectively.

AlloHSCT was performed in 28 (63\%) children in CR2, at median time of 2.5 months (range 0.2-5.5) from second

Table 2. Treatment follow-up in children with refAML after CR1 according to the consolidation with alloHSCT

$\begin{gathered}\text { AlloHSCT after CR1 } \\ (n=4)\end{gathered}$
$\begin{aligned} & \text { No alloHSCT after CR1 } \\ & (n=1)\end{aligned}$
Relapse
Survival in CR1
$\begin{aligned} & n=1,26 \text { months }^{*} \\ & (\text { range } 14.9-34.2)\end{aligned}$
*time from CR1 in months; ${ }^{* *}$ median time from CR1


Table 3. Treatment follow-up of relAML children in CR2, according to the consolidation with alloHSCT

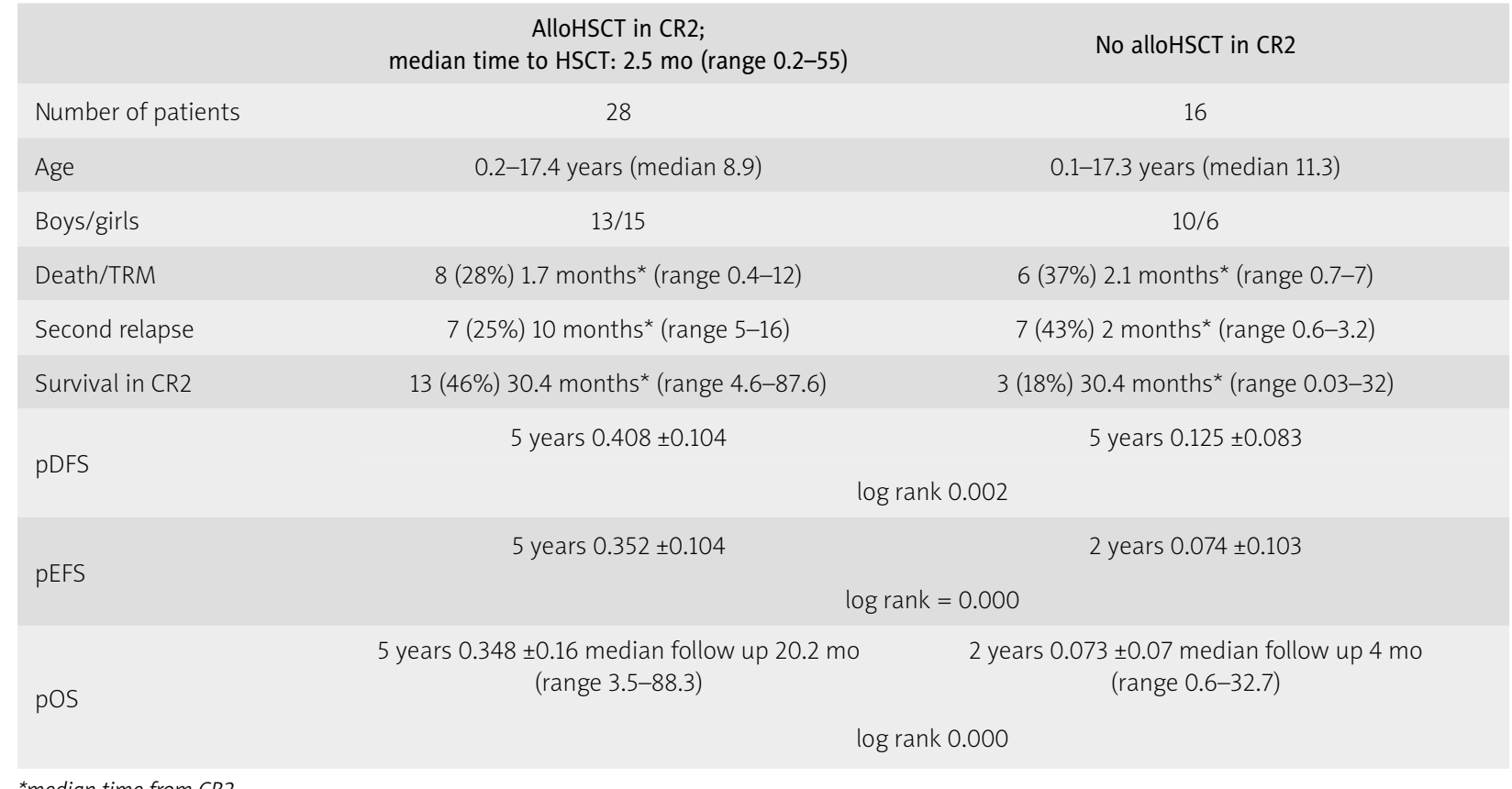

*median time from CR2

remission. Of 44 children in CR2 after reinduction chemotherapy, 16 (36\%) did not undergo alloHSCT. Table 3 shows the follow-up of the children in CR2, according to the subsequent alloHSCT therapy.

Among the transplanted children 8 died of toxic complications - 3 due to graft-versus-host disease, 2 due to multiorgan failure, and one each from sepsis, CNS hemorrhage, and lymphoproliferative disease. In 6 non-transplanted patients treatment-related mortality was caused by multiorgan failure, sepsis and CNS hemorrhage in 3, 2 and one case respectively.

Two children received MD alloHSCT in relapse resulting in one CR2 attained. Both of them died, one due to hemorrhagic complications in CR2, the other because of progressive disease, 47 and 37 days from alloHSCT respectively.

By December 31, 2011, 16 (35\%) of 62 relAML children, including 1 poor early responder ${ }^{1}$ and 3 non-grafted patients, were alive, with a median time from CR2 of 30.4 months (range 0.03-87.6). One of the non-transplanted survivors was a 17-year-old boy with SR AML FAB M4 inv16, with 2 years CR1 duration. The other one was a 2-year-old boy at diagnosis, with AML FAB M4E, delayed CR1 (HR), early CNS relapse, and a good response to one Flag course and CNS radiotherapy. The third one was a 15 -year-old boy with HR AML BCR/ABL positive (FAB data missing), late marrow relapse, and remission entry after IdaFlag plus dasatinib. The time of follow-up of these 3 boys was 0.6, 32 and 4 months respectively.

Table 4 shows the comparison of the therapy results in refAML and relAML.

\section{Prognostic factors}

The impact of selected prognostic factors (age, risk group, early response to chemotherapy, duration of CR1,
HSCT in relapse) for CR2 rate and survival was analyzed in the group of relAML.

Early relapse was significantly correlated with lower remission rates (62\% vs. $90 \% P=0.046$; Table 5 ) and both 5 -year pDFS $\left(P_{\text {log-rank }}=0.03\right)$ and pOS $\left(P_{\text {log-rank }}=0\right)$.

There was a marginally significant difference in overall survival between high and standard risk patients ( $P_{\text {log-rank }}$ $=0.035)$. A significant effect of alloHSCT on survival was identified in the log-rank test (pDFS: $P_{\text {log-rank }}=0.02$; pOS: $\left.P_{\text {log-rank }}=0\right)$.

In a Cox regression model using age, risk group, CR1 duration, achievement of CR2 and HSCT in relapse, only HSCT after CR2 showed a strong impact on survival (Tables 6 and 7; Fig. 1).

The variety of postreinduction chemotherapy regimens used in our group precludes the evaluation of the impact of any one therapeutic schedule.

\section{Discussion}

The 5 -year OS of $16 \%$ for children with relAML in this report reflects the poor prognosis for these patients. The survival rates in childhood relAML have increased over time with intensified and uniform reinduction concepts and improved supportive care. They range from $12 \%$ to $34 \%$ at 2-5 years in other studies [6] with recent relatively good outcome of $38 \%$ at 4 years in Relapsed BMF AML 2001/01 and $38 \%$ at 5 years in Nordic Society for Pediatric Hematology and Oncology (NOPHO) AML93 trials [3, 5]. Results presented here fit the lower range of these limits but the comparability may not be accurate due to methodological discrepancies between the trials.

Whereas the survival of the patients in the presented cohort was unsatisfactory, the second remission rate of $77 \%$ was comparable with those shown by the leading 
Table 4. Summary of outcome and follow-up of children with refAML and relAML

\begin{tabular}{|c|c|c|}
\hline & refAML $(n=16)$ & relAML $(n=62)$ \\
\hline $\begin{array}{l}\text { Postreinduction } \\
\text { CR rate }\end{array}$ & $n=5(33 \%)$ & $n=45(77 \%)$ \\
\hline First/second relapse & $n=2 / 5$ (0.3 and 26 months $)^{*}$ & $n=14 / 45(31 \%) 16.4$ months (range 0.6-16.4) \\
\hline Survival in CR1/CR2 & $n=3 / 533.2$ months (range 17.8-34.2) ${ }^{\star *}$ & $n=16 / 45(35 \%) 30.4$ months (range $0.03-87.6)^{\star *}$ \\
\hline pDFS & 2 years, $0.533 \pm 0.248$ & 5 years, $0.305 \pm 0.07$ \\
\hline pEFS & 3 years, $0.167 \pm 0.093$ & 5 years, $0.177 \pm 0.05$ \\
\hline pOS & $\begin{array}{c}3 \text { years, } 0.25 \pm 0.108 \\
\text { median follow-up } \\
37.3 \text { months (range 17.8-37.4) }\end{array}$ & $\begin{array}{c}5 \text { years, } 0.164 \pm 0.079 \\
\text { median follow-up } \\
19.8 \text { months (range } 0.6-88.3 \text { ) }\end{array}$ \\
\hline
\end{tabular}

groups: 77\% (NOPHO) [3], 71\% (French Leucémie Aique Myeloide Enfant 89/91 protocol-LAME) [4]. This observation can be explained with the low quality of the remissions and directs the attention toward the need for optimal chemotherapy choice. Prospective monitoring of minimal residual disease (MRD) is supposed to be a more accurate technique to evaluate the disease status than remission rate $[1,9]$.

In accordance with other reports, in the presented group the second remission rate and survival differed according to the first remission duration [3, 4, 7, 8, 13]. Early relapse was associated with a statistically significantly lower CR2 rate (62\% vs. $90 \%$ ) and worse 5 -year pOS (11\% vs. $27 \%$ ) than late relapse. Presented survival in early relapse was similar to 3-year pOS of 24\% in the MRC AML10 trial; however, NOPHO 93 and LAME 89/91 studies reported 21\% and $24 \%$ at 5 years.

The prognosis in the analyzed refAML group was dismal, with CR1 rate, disease-free survival (DFS), event-free survival (EFS) and overall survival (OS) of 33\%, 53\% at 2 years, $16 \%$ at 3 years and $25 \%$ at 3 years vs. $77 \%, 30 \%$ at 5 years, $17 \%$ at 5 years and $16 \%$ at 5 years in relAML respectively. In contrast to relAML, among the presented 16 children with refAML, no one had standard risk AML, the CR1 rate was half the level of the $C R 2$ rate in relAML, and the final prognosis was much worse. These findings show that probably refAML should be evaluated as a distinct entity.

There are few reports describing treatment outcomes in cohorts of children with refAML. Of 11 refAML patients in the DCOG study, no one attained CR1 [6]. Gorman et al. reported 2-year DFS of $0 \%$ in a group of 7 refAML children [10]. Due to the small numbers, these children are incorporated into cohorts of relAML or poor responder $\mathrm{AML}$ patients. Warenham et al. have just reported (NOPHO-AML 2004) the outcome of early alloHSCT in 14 refAML patients. Two of them were transplanted in persistent disease and 9 with positive MRD. These 11 patients became long-term survivors (3-year follow-up), recommending early alloHSCT regardless of the disease state in such cases [11].

In the presented ref/relAML cohort, patients who were transplanted in postreinduction remission had the best prognosis and accounted for $100 \%$ of refAML and $84 \%$ of relAML survivors. AlloHSCT in CR2 was statistically the leading predictor of better outcome in relAML children. This is consistent with the literature data $[3,4,7,10]$ and the documented graft versus leukemia effect which is the basis of the established consensus to advocate alloHSCT to all ref/relAML patients in postreinduction remission [1, 2]. This therapy gives the chance of 5 -year survival of up to $49-62 \%$ in different studies [12], compared with 35\% in the presented cohort. It shows that details of the transplant procedure, toxicities and supportive care modalities should be analyzed, which is planned to be done in a separate paper.

In the analyzed study the median time between CR2 and second relapse or treatment-related death in the non-transplanted patients was 2 and 2.1 months respectively. These time periods were shorter than the median time to transplant (2.5 months) in the grafted children. It suggests that these circumstances precluded HSCT and

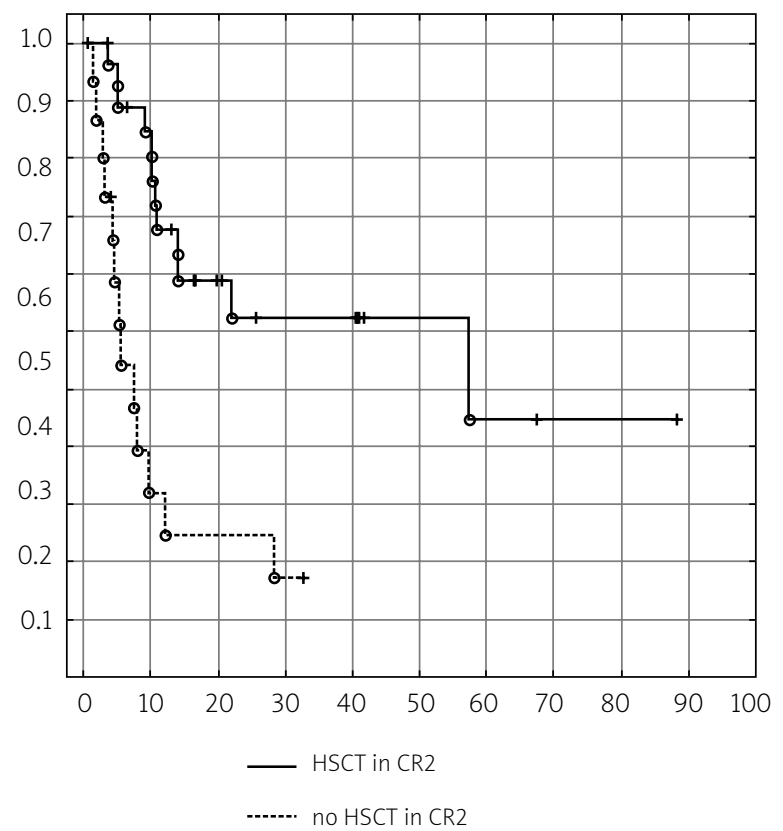

HSCT - hematopoietic stem cell transplantation

Fig. 1. Probability of survival according to consolidation in CR2 
Table 5. Evaluation of the impact of selected prognostic factors on CR2 rate in relAML children

\begin{tabular}{|c|c|c|c|c|c|}
\hline \multirow{2}{*}{ Factor } & & \multirow{2}{*}{$n$} & \multirow{2}{*}{ CR2 $(n=44)$} & \multicolumn{2}{|c|}{ Univariate analysis } \\
\hline & & & & $z$ & $p$ \\
\hline \multirow{2}{*}{ age } & $<10$ years & 27 & $70 \%$ & \multirow{2}{*}{0.205106} & \multirow{2}{*}{0.837489} \\
\hline & $>10$ years & 35 & $74 \%$ & & \\
\hline \multirow{2}{*}{ risk group } & $S R$ & 17 & $94 \%$ & \multirow{2}{*}{1.51033} & \multirow{2}{*}{0.130960} \\
\hline & $\mathrm{HR}$ & 45 & $64 \%$ & & \\
\hline \multirow{2}{*}{ early response } & $<20 \%$ & 37 & $83 \%$ & \multirow{2}{*}{0.223753} & \multirow{2}{*}{0.822950} \\
\hline & $>20 \%$ & 7 & $71 \%$ & & \\
\hline \multirow{2}{*}{ CR1 duration } & $\leq 1$ year & 40 & $62 \%$ & \multirow{2}{*}{1.99513} & \multirow{2}{*}{0.046030} \\
\hline & $>1$ year & 22 & $90 \%$ & & \\
\hline
\end{tabular}

Table 6. Evaluation of the impact of selected prognostic factors on DFS in relAML children

\begin{tabular}{|c|c|c|c|c|c|c|}
\hline \multirow{2}{*}{ Factor } & & \multirow{2}{*}{$N$} & \multirow{2}{*}{ pDFS } & \multirow{2}{*}{ log-rank } & \multicolumn{2}{|c|}{ Cox regression } \\
\hline & & & & & HR $(95 \% \mathrm{Cl})$ & $p$ \\
\hline age & $\begin{array}{l}<10 \text { years } \\
\geq 10 \text { years }\end{array}$ & $\begin{array}{l}19 \\
26\end{array}$ & $\begin{array}{l}5 \text { years, } 0.307 \pm 0.113 \\
5 \text { years, } 0.305 \pm 0.094\end{array}$ & 0.709 & 1.578 & 0.323692 \\
\hline risk group & $\begin{array}{l}\mathrm{SR} \\
\mathrm{HR}\end{array}$ & $\begin{array}{l}16 \\
34\end{array}$ & $\begin{array}{l}5 \text { years, } 0.499 \pm 0.13 \\
5 \text { years, } 0.218 \pm 0.078\end{array}$ & 0.153 & 2.328 & 0.083611 \\
\hline CR1 length & $\begin{array}{l}\leq 1 \text { year } \\
>1 \text { year }\end{array}$ & $\begin{array}{l}25 \\
20\end{array}$ & $\begin{array}{c}5 \text { years, } 0.24 \pm 0.08 \\
5 \text { years, } 0.382 \pm 0.122\end{array}$ & 0.030 & 0.465 & 0.104055 \\
\hline HSCT after CR2 & $\begin{array}{c}\text { HSCT } \\
\text { no HSCT }\end{array}$ & $\begin{array}{l}28 \\
16\end{array}$ & $\begin{array}{l}5 \text { years, } 0.408 \pm 0.083 \\
2 \text { years, } 0.125 \pm 0.083\end{array}$ & 0.002 & 2.751 & 0.01458 \\
\hline
\end{tabular}

Table 7. Evaluation of the impact of selected prognostic factors on OS in relAML children

\begin{tabular}{|c|c|c|c|c|c|c|}
\hline \multirow{2}{*}{ Factor } & & \multirow{2}{*}{$n$} & \multirow{2}{*}{ pOS } & \multirow{2}{*}{ log-rank } & \multicolumn{2}{|c|}{ Cox regression } \\
\hline & & & & & HR $(95 \% \mathrm{Cl})$ & $p$ \\
\hline \multirow{2}{*}{ age } & $<10$ years & 27 & 5 years, $0.258 \pm 0.096$ & \multirow{2}{*}{0.502} & \multirow{2}{*}{1.962} & \multirow{2}{*}{0.057} \\
\hline & $\geq 10$ years & 35 & 5 years, $0.121 \pm 0.095$ & & & \\
\hline \multirow{2}{*}{ risk group } & $S R$ & 17 & 5 years, $0.238 \pm 0.184$ & \multirow{2}{*}{0.035} & \multirow{2}{*}{2.119} & \multirow{2}{*}{0.090} \\
\hline & $H R$ & 45 & 5 years, $0.182 \pm 0.063$ & & & \\
\hline \multirow{2}{*}{ CR1 length } & $<1$ year & 40 & 5 years, $0.119 \pm 0.057$ & \multirow{2}{*}{0.000} & \multirow{2}{*}{1.44} & \multirow{2}{*}{0.467} \\
\hline & $\geq 1$ year & 22 & 5 years, $0.272 \pm 0.202$ & & & \\
\hline \multirow{2}{*}{ CR2 } & CR2 & 45 & 5 years, $0.228 \pm 0.108$ & \multirow{2}{*}{0.000} & \multirow{2}{*}{5.326} & \multirow{2}{*}{0.000} \\
\hline & no CR2 & 17 & 0.48 year $0 \pm 0$ & & & \\
\hline \multirow{2}{*}{ HSCT } & $\mathrm{HSCT}$ & 30 & 5 years, $0.324 \pm 0.150$ & \multirow{2}{*}{0.000} & \multirow{2}{*}{2.989} & \multirow{2}{*}{0.024} \\
\hline & no HSCT & 32 & 2 years, $0.070 \pm 0.047$ & & & \\
\hline
\end{tabular}

shows the need for optimal care to avoid delay in proceeding to transplant.

The question may be raised whether further relapses are due to delay of HSCT or to a more aggressive type of leukemia or suboptimal first line treatment. On the other hand, favorable long-term outcomes after chemotherapy alone have also been reported. Goemans et al. in 2008 summarized from the literature 34 of them [6]. In our cohort there was one survivor with 32 months of follow-up after chemotherapy alone.

Genetic profiling and MRD monitoring are currently the modalities which, if performed after primary diagnosis, might provide insight into these doubts concerning AML heterogeneity. Specific genetic predictors and MRD strongly correlate with the treatment outcome [1, 2, 9] and may guide risk-stratified therapeutic decisions. The impact of the genetic characteristics on the management individualization was shown in the Relapsed AML 2001/01 trial. The patients with core binding factor acute myeloid leukemia (CBF-AML) treated with DaunoFlag had an OS of $82 \%$ at 4 years in contrast to $58 \%$ in those treated with FLAG only [5]. This risk-directed strategy may also be extended to the frontline AML therapy as it is proposed in the AML-BFM 2012 study: whereas HSCT in children in CR1 has been considered controversial so far, in this trial it is offered to subgroups with unfavorable genetics, assuming relapse rate reduction $[1,13]$.

In our nationwide study none of the refAML patients and nearly none of relAML ones presented the potential to survive a long time without HSCT in postreinduction 
remission. The key issue of current management of ref/ relAML is the reinduction regimen to achieve and maintain remission and optimal care to support the patient for early HSCT using the best genomic typing technology to select the best matched available donor [1, 2]. There is no standard reinduction chemotherapy protocol $[1,2]$. The concern should focus on the optimal balance between efficacy and toxicity of the chosen regimen and the unified rules of the therapy which allow to derive conclusions on future solutions. The development of highly sensitive MRD techniques standardized for all patients, genetic profiling and identification of other predictors are required for future more individualized treatment.

\section{Authors declare no conflict of interest.}

\section{References}

1. Rubnitz JE, Inaba H. Childhood acute myeloid leukaemia. Br J Haematol 2012; 159: 259-76.

2. Reaman GH, Smith FO. Childhood leukemia. Springer, Berlin Heidelberg 2011

3. Abrahamsson J, Clausen N, Gustafsson G, et al. Improved outcome after relapse in children with acute myeloid leukaemia. $\mathrm{Br} J \mathrm{Hae}$ matol 2007; 136: 229-36.

4. Aladjidi N, Auvrignon A, Leblanc T, et al. Outcome in children with relapsed acute myeloid leukemia after initial treatment with the French Leucemie Aique Myeloide Enfant (LAME) 89/91 protocol of the French Society of Pediatric Hematology and Immunology. J Clin Oncol 2003; 21: 4377-85.

5. Kaspers GJ, Zimmermann M, Reinhardt D, et al. Improved outcome in pediatric relapsed acute myeloid leukemia: results of a randomized trial on liposomal daunorubicin by the International BFM Study Group. J Clin Oncol 2013; 31: 599-607.

6. Goemans BF, Tamminga RY, Corbijn CM, Hählen K, Kaspers GJ. Outcome for children with relapsed acute myeloid leukemia in the Netherlands following initial treatment between 1980 and 1998 survival after chemotherapy only? Haematologica 2008; 93: 1418 20.

7. Rubnitz JE, Razzouk BI, Lensing S, Pounds S, Pui CH, Ribeiro RC. Prognostic factors and outcome of recurrence in childhood acute myeloid leukemia. Cancer 2007; 109: 157-63.

8. Webb DK, Wheatley K, Harrison G, Stevens RF, Hann IM. Outcome for children with relapsed acute myeloid leukaemia following initial therapy in the Medical Research Council (MRC) AML 10 trial. MRC Childhood Leukaemia Working Party. Leukemia 1999; 13: 25-31.

9. van der Velden VH, van der Sluijs-Geling A, Gibson BE, et al. Clinical significance of flowcytometric minimal residual disease detection in pediatric acute myeloid leukemia patients treated accord ing to the DCOG AnLL97/MRC AML12 protocol. Leukemia 2010; 24: 1599-606.

10. Gorman MF, Ji L, Ko RH, et al. Outcome for children treated for relapsed or refractory acute myelogenous leukemia (rAML): a Therapeutic Advances in Childhood Leukemia (TACL) Consortium study. Pediatr Blood Cancer 2010; 55: 421-9.

11. Wareham NE, Heilmann C, Abrahamsson J, et al. Outcome of poor response paediatric AML using early SCT. Eur J Haematol 2013; 90: 187-94.

12. Sander A, Zimmermann M, Dworzak M, Fleischhack G, von Neuhoff C, Reinhardt D, Kaspers GJ, Creutzig U. Consequent and intensified relapse therapy improved survival in pediatric $A M L$ : results of relapse treatment in 379 patients of three consecutive AML-BFM trials. Leukemia 2010; 24: 1422-8.

13. AML-BFM 2012 of Acute Myeloid Leukemias in children and adolescents.

\section{Address for correspondence}

Jolanta Skalska-Sadowska MD, PhD

Department of Pediatric Hematology,

Oncology and Transplantology

Poznan University of Medical Sciences

Szpitalna 27/44

60-572 Poznań, Poland

tel. +48618491447

$\mathrm{fax}+48618474356$

e-mail: jsk@poczta.onet.eu

Submitted: 1.09.2013

Accepted: $\quad 16.10 .2013$ 